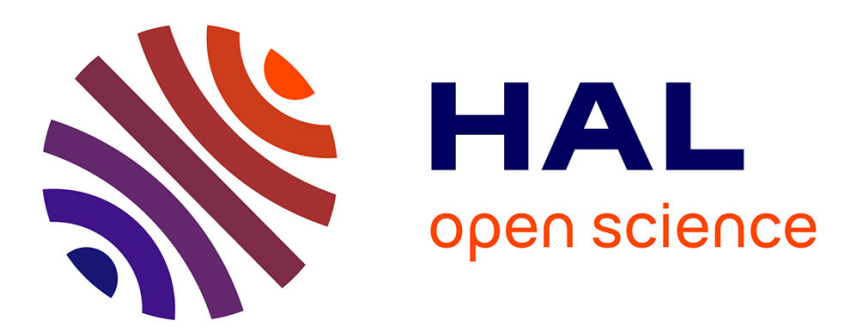

\title{
Direct duplication of physical models in discrete 5-axis machining
}

Almohanad Makki, Claire Lartigue, Christophe Tournier, François Thiebaut

\section{To cite this version:}

Almohanad Makki, Claire Lartigue, Christophe Tournier, François Thiebaut. Direct duplication of physical models in discrete 5-axis machining. Virtual and Physical Prototyping, 2008, 3 (2), pp.93-103. 10.1080/17452750802047941 . hal-00604250

\section{HAL Id: hal-00604250 \\ https://hal.science/hal-00604250}

Submitted on 28 Jun 2011

HAL is a multi-disciplinary open access archive for the deposit and dissemination of scientific research documents, whether they are published or not. The documents may come from teaching and research institutions in France or abroad, or from public or private research centers.
L'archive ouverte pluridisciplinaire HAL, est destinée au dépôt et à la diffusion de documents scientifiques de niveau recherche, publiés ou non, émanant des établissements d'enseignement et de recherche français ou étrangers, des laboratoires publics ou privés. 


\title{
Direct duplication of physical models in discrete 5-axis machining
}

\author{
M. Makki ${ }^{\mathrm{a}}$, C. Lartigue ${ }^{\mathrm{a}, \mathrm{b}^{*}}$, C. Tournier ${ }^{\mathrm{a}}, \mathrm{F}$. Thiébaut ${ }^{\mathrm{a}, \mathrm{b}}$ \\ ${ }^{a}$ LURPA, ENS de Cachan ; ${ }^{b}$ IUT de Cachan, Université Paris 11, Cachan, France
}

\begin{abstract}
This paper deals with the feasibility of direct duplication of physical objects as regards a classical approach based on Reverse Engineering. The object surface acquisition is performed using optical devices. Algorithms for tool path generation directly from the cloud of points have been developed in 3-axis machining. A n-buffer simulation completed by the real machining emphasize that direct duplication is efficient in terms of conformity to the geometry. As the surface reconstruction step is removed, the proposed process is faster and simple to implement. The last step is concerned with the extension to discrete 5-axis machining. The main issue is related to the determination of the part set-ups and the corresponding point cloud segmentation. Keyword : Tool path generation, Area-by-area machining, Point cloud Segmentation
\end{abstract}

\section{$1 \quad$ Introduction}

Physical object duplication is nowadays an important issue for it permits to recreate hand-made objects or prototypes. Most methods for physical object duplication rely on Reverse Engineering (RE). The process basically consists of three majors independent steps: (1) data acquisition using 3D digitizing; (2) CAD model construction from the data points and (3) $\mathrm{NC}$ tool path generation from the CAD model (OuYang et al., 2005).

The step of data acquisition consists of measuring the physical object using non contact digitizing systems such as 3D laser scanners. This generally provides a large cloud of points representative of the object's surfaces. The process of converting data points into a usable CAD model (also well-known as RE) includes various phases: data pre-processing, data segmentation, data fitting, and final model construction (Varady et al., 1997). Despite the advances in CAD modelling of discrete data and the new RE software systems now available, this step is seldom automatic. It is generally time-consuming, tedious and the quality of the resulting surface strongly depends on the possibilities offered by the software, in particular in the area of segmentation and precision surface fitting for complex objects (Feng and Teng, 2005). The obtained CAD model thus constitutes an approximation, more or less

\footnotetext{
* Corresponding author : lartigue@lurpa.ens-cachan.fr
} 
correct, of the digitized data. Concerning NC tool path generation, many robust methods exist for continuous surfaces applicable in 3-axis machining as well as in 5-axis machining. However, as the calculated tool path is also an approximation of the CAD model, the combined procedure suffers from error accumulation and inefficiency (Feng and Teng, 2005).

As the stage of RE is a restraint to automation and precision of the process, recent research has addressed the issue of direct duplication. Direct duplication consists in generating tool path machining directly from data points. In the last decade, several investigations have been made in this direction. The first method reported is the work by Lin and Liu (Lin and Liu, 1998). Authors create a rectangular mesh, a Z-map, from the digitizing lines obtained with contact or non-contact measuring devices. The rectangular mesh is oriented according to the driving measuring direction, and is the support for tool path generation. Park and Chung (Park and Chung, 2003) proposed a method for both roughing and finishing. Rough machining is performed slice by slice. Taking advantages of the digitizing lines, authors defined finishing tool paths as a series of point sequence curves. When the collected points form an inhomogeneous cloud of data, their algorithms can not be applied. Feng and Teng (Feng and Teng, 2005) present a method to generate tool path for which machining error and surface finish are explicitly considered. A CL-net (Cutter Location net) is created which consists of 2D cells sequentially ordered. The size of the cell net is defined relatively to the machining tolerance (for its length) and the maximum scallop height allowed (for its side size). Each cell node corresponds to a CL point. Due to the absence of continuity, authors evaluate machining errors based on two adjacent tool swept surfaces of the ball-end cutter tool. An optimization method of the position of the cell node is thus proposed to minimize the machining error. More recently, Teng et al (Teng et al., 2006) introduced the concept of area by area machining of complex parts in order to increase machining efficiency. The point cloud is segmented in function of the geometric complexity of points. The latter is defined considering local curvature, and possible gouging. Therefore, points with the same complexity are grouped together to form distinct machining areas. Tool paths are generated for each machining area using the method developed in authors' previous work (Feng and Teng, 2005). However, the point cloud proposed for illustration comes from the sampling of a CAD model, and not a real part digitizing. Peng and Ying (Peng and Yin, 2007) have handled the issue of tool path regeneration if a modification in the design process must be taken into account. The authors assumed that the original tool path is generated from B-spline surfaces constructed by selecting some measured points as control points. With the base of Bspline surfaces, only modified regions are identified and corresponding tool paths are easily regenerated. In their work, Ou Yang et al (Ou Yang et al, 2005) exposed the algorithm they have developed to automatically select an optimized cutter-tool size for 3-axis ball end milling of a point 
cloud. Algorithm efficiency was merely tested using simulated point cloud data sets. Concerning 5axis machining, the approach developed in (Chui et al, 2008) is interesting. Authors based their method on a 3D triangular mesh construction directly from the cloud of points. For each facet, the local normal is following obtained using the facet and its neighbours. The axis tool is thus oriented from the normal using a value of the tilt angle set by the user.

Therefore, literature mentions little work for tool-path generation on large clouds of points obtained by the digitizing according to various sensor configurations. Indeed, in this case, collected points form a large cloud of points, non-ordered, dense, noisy, and inhomogeneous. Moreover, few approaches address the problem of discrete 5-axis machining directly from the cloud of points without surface or meshing reconstruction. For each part set-up, the portion of surface accessible by the tool (most generally a ball-end cutter tool) is machined using 3-axis machining algorithms. The issue is to find the minimal number of set-ups allowing the whole surface to be machined.

With the objective to machine large point clouds, dense, inhomogeneous, noisy and incomplete, we have proposed algorithms for tool path generation that evolved from 3-axis machining (Osty et al. 2000) to discrete 5-axis machining (Breteau et al. 2006). The paper deals with the presentation of those algorithms in the context of direct duplication of physical objects. Hence, a specific attention is given to show the efficiency of direct duplication as regards classical methods of duplication based on surface reconstruction (RE). The paper is organized as follows. Section 2 presents algorithms of tool path generation in 3 axes from massive data points, integrating both the roughing and finishing operations. No meshing or continuous surface construction is necessary. An illustration of direct duplication of a complex physical object is exposed. A comparison is done with a classical method of duplication relying on RE. Section 3 concerns the extension to 5-axis direct duplication. As discrete machining is used, the first step aims at finding points that can be machined with the same tool orientation, defining thus a part set-up. This involves determining tool visibility and accessibility. The following step concerns the point cloud segmentation into areas, each one corresponding to a part setup. Each area is machined in 3 axes using algorithms exposed in section 2. The application proposed in section 4 highlights issues that required specific developments and optimization. The paper is ended by some conclusions. 


\section{Tool path generation in 3-axis ball end-milling for direct duplication}

As the objective is to produce a copy of an existing object, based on data points collected on the physical object surfaces, the main issue is tool path generation directly from discrete data, including both roughing and finishing milling operations. Although there are many methods for tool path finishing trajectories, a few ones address the specific case of roughing. Lin and Liu (Lin and Liu, 1998) have proposed a method based on the classical Z-level roughing method. In order to overcome, difficulties linked to the huge amount of data, and the lack of continuity their work relies on a structure of the data through a Z-map calculated from the digitizing lines. Hence, to be efficient, algorithms for tool path generation requires a structure of the data.

In the present section, we propose a methodology for 3-axis milling duplication which encompasses roughing and finishing. For both milling operations, algorithms developed take advantages of a voxelisation of the data, which consists in splitting up the cloud points into cells, so that each cell contains a minimum of points. As only non-empty cells (or voxels) are considered, attributes linked to points included in the space of the voxel can be calculated, and associated to each voxel. The barycentre of the points and the normal to the least-square plane that best-fits the points included in the voxel are the most useful attributes for tool path generation. Therefore, voxelisation gives data structure, pseudo-continuity and a lightened representation which will facilitate further calculations.

\subsection{Roughing}

The Z-level strategy is adopted, for which the roughing is performed layer-by-layer, in a direction perpendicular to the Z-axis tool. In each level (or layer) $Z i$, the machining mode is the "zig-zag" mode, and the trajectory $T i$ is calculated according to the roughing parameters (Figure 1). 


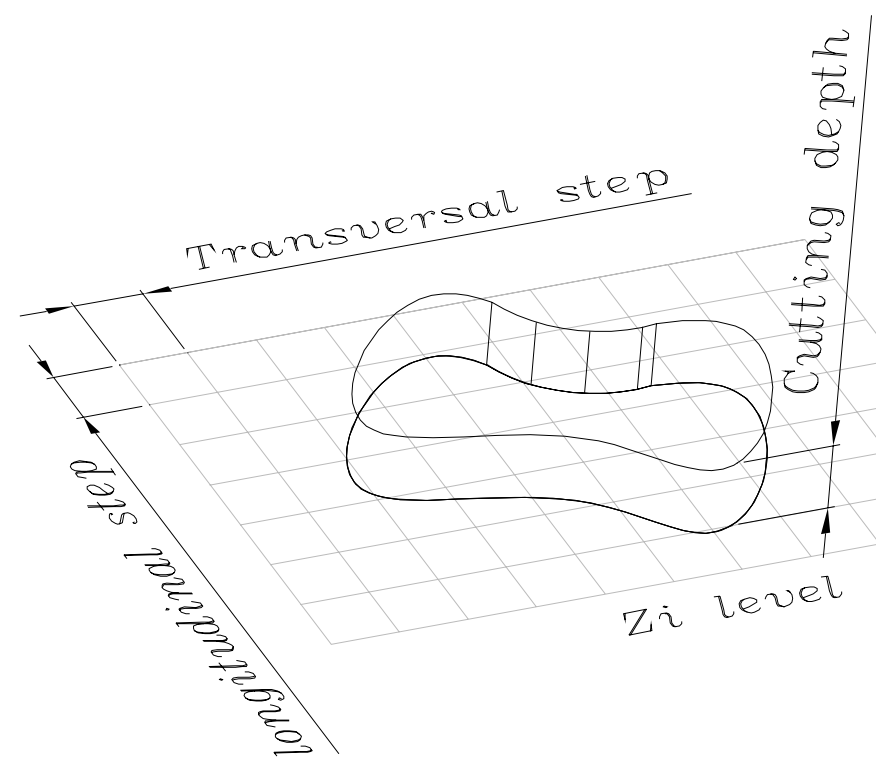

Figure1. Roughing strategy

For this purpose, the voxelisation of the data is carried out as regards those parameters. The voxel height corresponds to the cutting depth. In each layer, a grid $(\mathrm{X}, \mathrm{Y})$ is built. The longitudinal step is defined in the feed rate direction whereas the transversal step corresponds to the step over. Those parameters provide the quality of the milled surface as regards machining time. Grid nodes $n_{i j}$ are organized in function of the sweeping direction adopted. For each node $n_{i j}$, interferences between the tool and the barycentre of the neighbour voxels are checked. Nodes which are interference free define points of the trajectory $T i$ for the layer $Z i$. For each point in interference, an off-machining trajectory is inserted to avoid collision with the part (Figure 2).

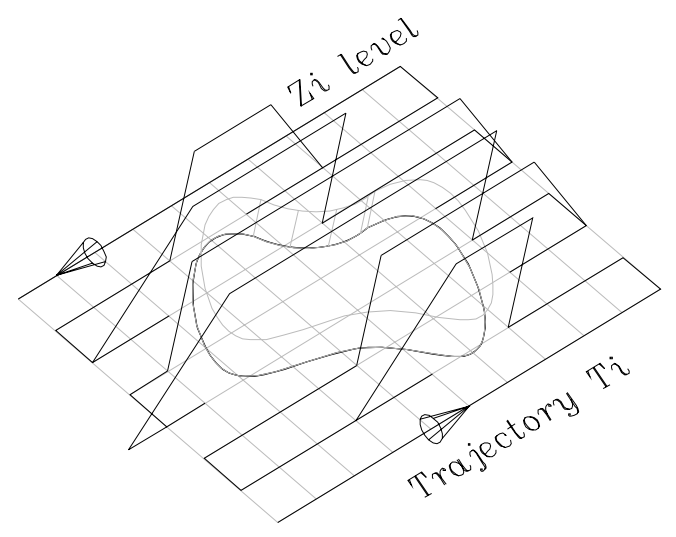

Figure 2. Resulting roughing trajectory 
The link between trajectories is ensured thanks to off-machining trajectories. To avoid tool damages, axial plunges are forbidden, and the tool must follow a ramp (Figure 3): the tool plunges down to the level $Z_{i-1}$, then it follows a slope down to the level $Z i$.

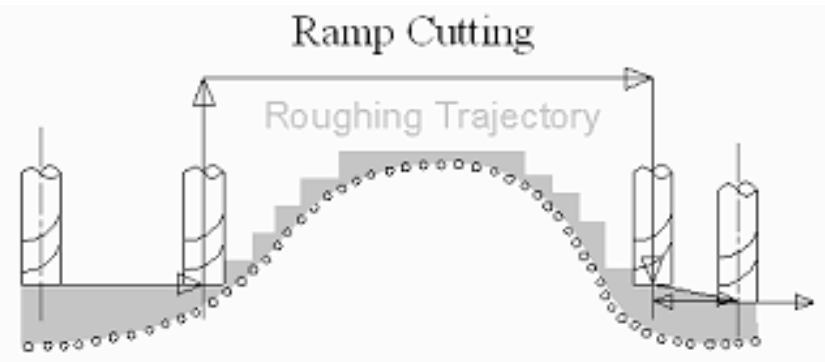

Figure 3. Ramp mode approach

The previously described algorithm has been implemented using Matlab. As the verification of interferences are not yet managed for ramp mode approach, only the machining of part without internal pockets is allowed, which is convenient for the complex free-form parts tested. An illustration will be proposed in section 2.3 .

\subsection{Finishing}

The finishing operation is a classical 3-axis ball-end milling. The method we have developed for tool path generation relies on the z-buffer inverse method (Suzuki et al, 1991). As for roughing, a grid in the xy-plane is built the dimensions of which are linked to the classical machining parameters, the machining tolerance $M t$, and the maximum scallop height allowed, $\mathrm{hm}$. The ordering of the grid nodes is processed according to the machining strategy. Let $\mathrm{R}$ be the tool radius, for each grid node $K_{i j}$, a tool positioning is calculated as follows (Figure 4) (Breteau et al, 2006):

1. Collect the points $P^{k}$ belonging to the point cloud that are included in a cylinder the radius of which is $\mathrm{R}$, and which axis is the $\mathrm{Z}$-axis passing through the considered node.

2. For each point $P^{k}$, calculate the intersection $\mathrm{Z}^{\mathrm{k}}$ between the $\mathrm{Z}$-axis and a sphere of radius $\mathrm{R}$, centred at the point.

3. Tool positioning $C L_{i j}$ is defined by the maximal $\mathrm{Z}^{\mathrm{k}}$ value. 

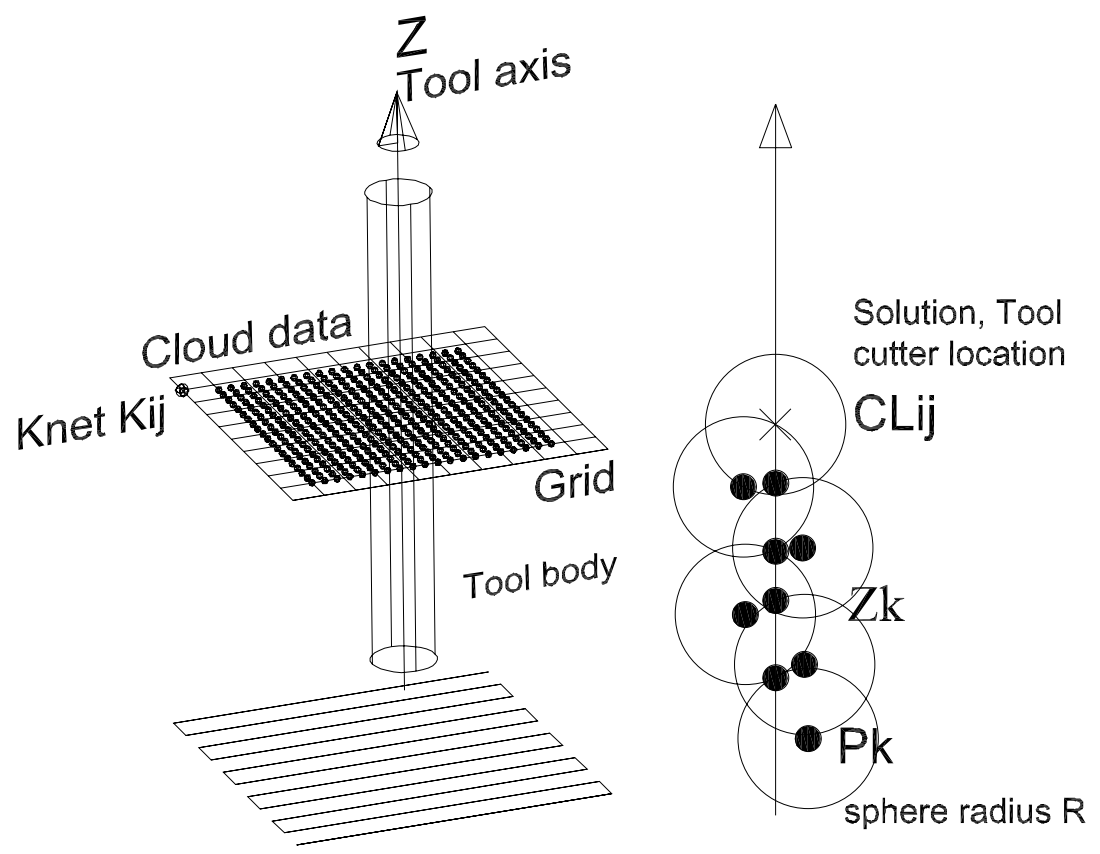

Figure 4. Finishing strategy

For the first implementations, grid parameters are chosen uniform; the final result is thus not optimized as regards part quality. Indeed, in order to avoid facets, the grid parameter is determined in function of the most discriminating areas of the point cloud as regards the chord deviation criterion. This involves over-quality for other portions. To overcome this problem, a solution should be the use of an adaptive longitudinal step. In such a case, a maximum distance is imposed between two successive points of the trajectory instead of two successive nodes of the grid. The integration of this solution is presently in progress.

\subsection{Comparison with classical methods based on RE}

In this section, we propose to compare the efficiency of direct duplication with regard to a classical approach based on RE. The comparison aims to assess the relevance of direct duplication vis à vis the classical RE methods, especially in terms of rapidity and fidelity.

A mask, the CAD model of which is unknown, is first digitized using a laser-scanner mounted on a CMM. Three different orientations are used leading to 470000 points. The completeness is ensured by using an automated scan planning strategy (Mehdi-Souzani et al, 2006). In order to lighten the point 
cloud, a filtering operation based on the chord error is applied, leading to 170000 points (Figure 5a). Both approaches are thus conducted in parallel.

For the RE method, the surface is reconstructed using the module Quick Surface Reconstruction of CATIA V5®. The point cloud is split into sub-sets to which a patch is associated. All the patches are therefore linked together, and remaining holes are filled so that the final surface is at least $\mathrm{C} 0$ continuous (Figure 5b). In order to decrease processing time, a scaling factor of $7 / 20$ is applied to the surface. Tool paths are afterwards generated from this surface using the module Advanced Machining of CATIA V5®. Roughing is performed starting from a raw material, which dimensions are: $100 \mathrm{~mm} \times 100 \mathrm{~mm} \times 60 \mathrm{~mm}$, and using a cylindrical tool the radius of which is $D_{R}=10 \mathrm{~mm}$. The finishing stage is carried out according to the parallel plane strategy. Machining parameters are reported in Table 1.

\begin{tabular}{|l|l|l|l|}
\hline Tool diameter: & Machining tolerance: & Feedrate: & Max. Scallop height: \\
$D=8 \mathrm{~mm}$ & $M t=0.01 \mathrm{~mm}$ & $V f=5 \mathrm{~m} / \mathrm{min}$ & $h m=0.01 \mathrm{~mm}$ \\
\hline
\end{tabular}

Table1. Machining parameters

The part machining is carried out on the milling centre of the laboratory: Mikron UCP 710 equipped with a $\mathrm{CN}$ unit Siemens 840D. In order to smooth trajectories, a real-time polynomial interpolation is applied with a tolerance of $0.01 \mathrm{~mm}$.

As direct duplication is concerned, the same scaling factor is applied to the point cloud. Tool paths are generated using the algorithms described above. Tool diameters are identical to those used for the RE method. For the roughing operation, the voxel size is chosen equal to $2 \mathrm{~mm}$, which defines the cutting depth and the longitudinal step. As the tool diameter is $D_{R}=10 \mathrm{~mm}$, the step over is given by $D_{R} / 2$. In order to avoid possible tool breaking and to uniform the material remaining before finishing, a semifinishing operation is performed. Trajectories for semi-finishing are the same that for finishing except that the tool used during machining is chosen of a smaller value $(\mathrm{D}=6 \mathrm{~mm})$, which leads to a uniform thickness of $1 \mathrm{~mm}$. For the finishing operation, grid steps are chosen identical for both $\mathrm{x}$ and $\mathrm{y}$ directions: $0.2 \mathrm{mmx} 0.2 \mathrm{~mm}$. To check that trajectories are interference free, they are integrated into CATIA V5®, and the simulation of the machining is carried out (Figure 5c). Algorithms for trajectory simulations based on the voxel-map are presently in progress.

The part is thus machined on the Mikron milling centre with the same feed rate. The result reported in figure $5 \mathrm{c}$ shows the feasibility of the approach. However, it is necessary to assess direct duplication in terms of geometrical conformity to the initial shapes. For this purpose, a comparison is made through simulations. 
The method developed is a classical N-buffer simulation method, and consists in, considering finishing only, simulating the envelope of the tool trajectory (Jerard et al. 1987). Geometrical deviations between the envelope and the initial cloud of points are calculated for both approaches (Classical RE and direct duplication). N-buffer requires a representation point/surface of the surface to be machined. The CAD model is used as the reference to calculate the normal at each cloud point. Despite imperfections introduced using such approximation, the default in normal orientation is of little influence on the geometrical conformity. Once the surfel (set of points and normals) is obtained, simulation is carried out by intersecting the surfel with the tool trajectory.
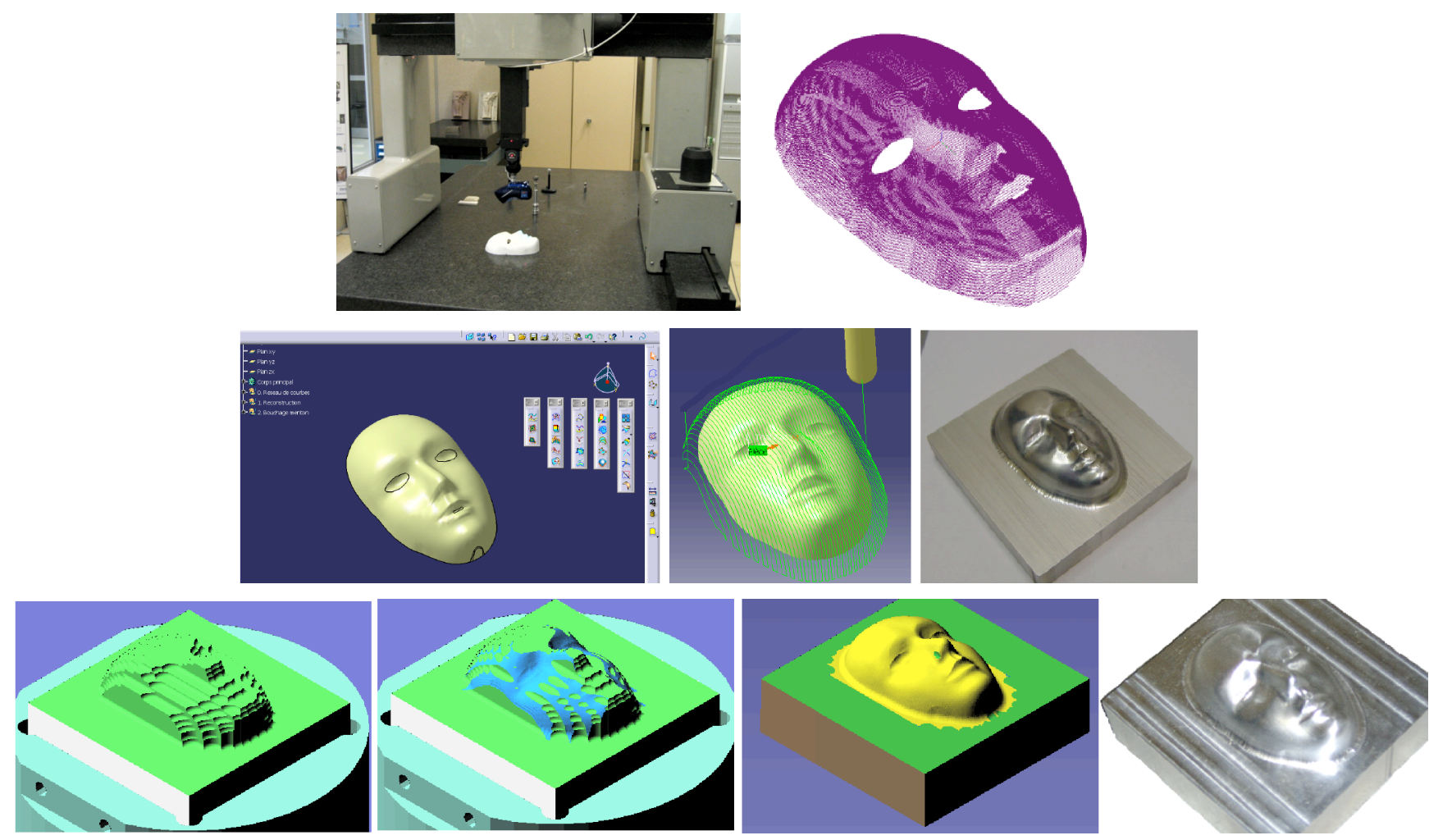

Figure 5. Duplication of a mask using RE and using direct duplication

a - Digitizing system and resulting point cloud

b - Method based on Reverse Engineering

c - Direct duplication

The algorithm is applied on a lightened cloud points (20000 points) (Figure 6). In the case of direct duplication, geometrical deviations reported are nearly all positive, which is consistent with the tool positioning method.. Details of the physical object are revealed: deformation of the left cheek, marks on the temples, inferior lip well-drawn, (Figure 7). Therefore, direct duplication quality is essentially linked to tool accessibility (tool diameter) and to the grid step. Indeed, deviations are negative only for vertical zones located near the chin for which the longitudinal step is too large. 
Similarly, the large transversal step provides important cups for the vertical zones at the vicinity of the temples. Concerning the approach based on RE, deviations are both positive and negative due to the surface reconstruction: the surface best-fits the points according to the least-square criterion. This provides waves as well as the smoothing of the details, which is particularly noticeable for the nose and the lips (Figures 6 and 7). The geometrical conformity is thus weaker. Nonetheless, the machining tolerance and the maximum scallop height are better managed with the software.
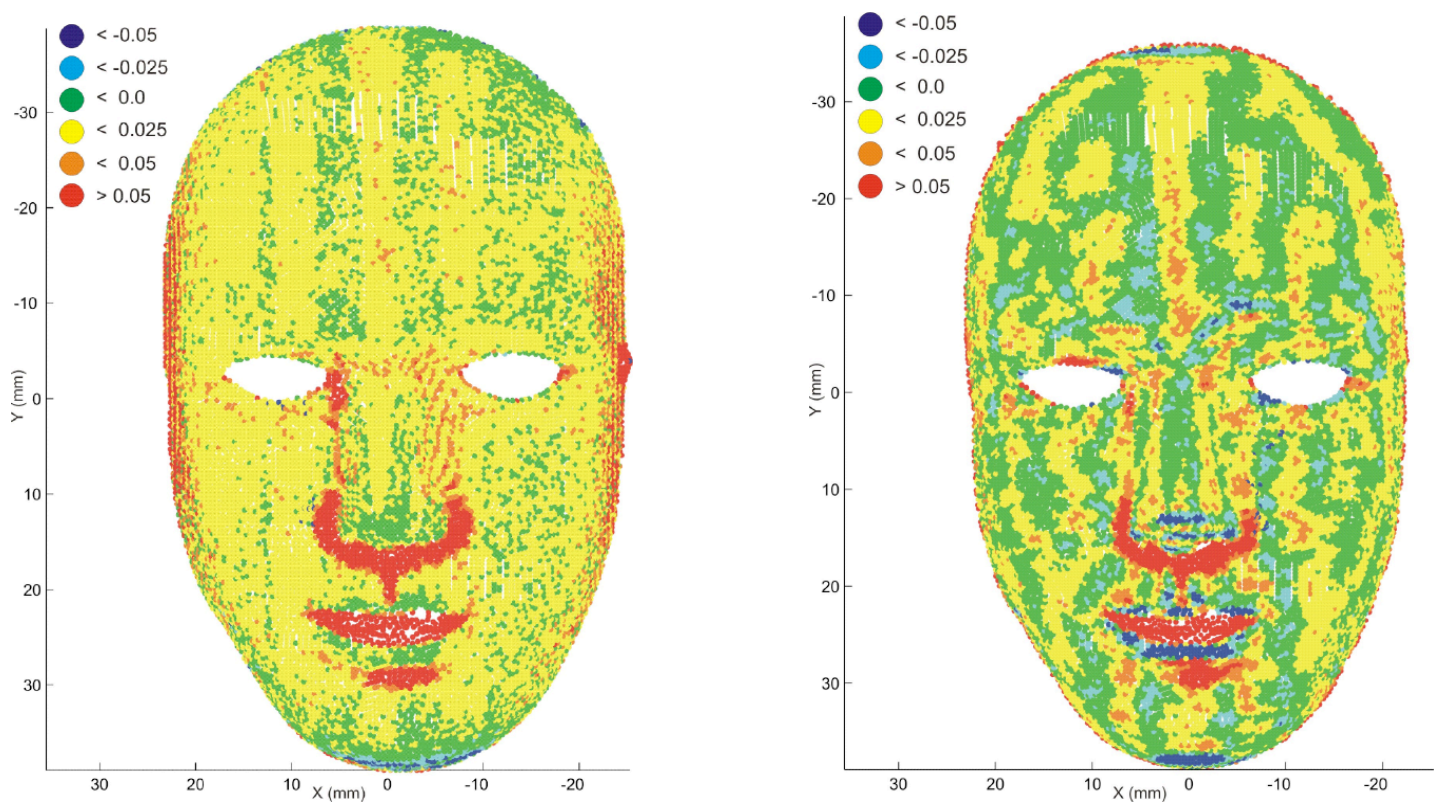

Figure 6. Comparison of machining simulations

a. Direct duplication, b. Reverse Engineering

The efficiency of direct duplication is proved. However, 3-axis machining is not capable of produced parts with complex shapes, such as statues for instance. An alternative is the use of 5-axis machining. The two additional degrees of freedom, allows the machining of sculptured surfaces with complex shapes. As some difficulties remain in interference-free tool path calculation for 5-axis machining, an alternative is the use of discrete 5-axis machining. In this approach, the surface to be machined is subdivided into areas with similar shapes, so that each area can be machined with the same tool orientation (Chen et al, 2003). A valid tool orientation defines a part set-up for which the surface can be machined without gouging and interference. Therefore, the area accessible by the tool can be machined according to 3-axis strategy.

In the next section, we propose an extension of 3-axis duplication to 5-axis direct duplication in order to increase the diversity of objects that can be reproduced. 

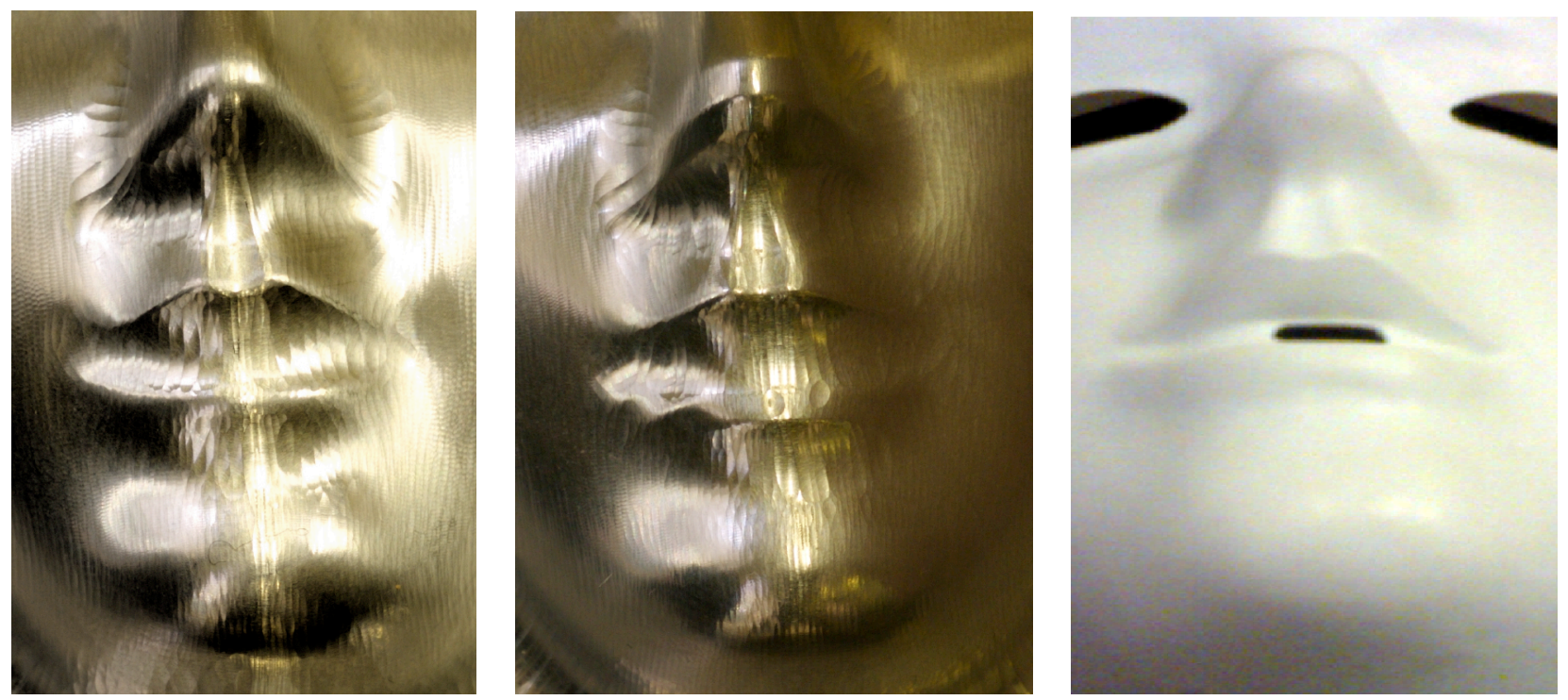

Figure 7. Details of the machined parts

a. Direct duplication, b. Reverse Engineering, c. Physical model

\section{$3 \quad$ From 3-axis copying to 5-axis Direct Copying}

Basically, 5-axis NC machining is an advanced machining technology, by which geometrically complex shapes can be machined. Thanks to the two additional axes, more generally rotational axes, the tool accessibility is increased. Therefore, for each point the number of tool accessibility orientations is nearly infinite. On the other hand, a minimum number of orientations (part set-ups) is required in order to completely and efficiently machine the part on the 5-axis machine tool.
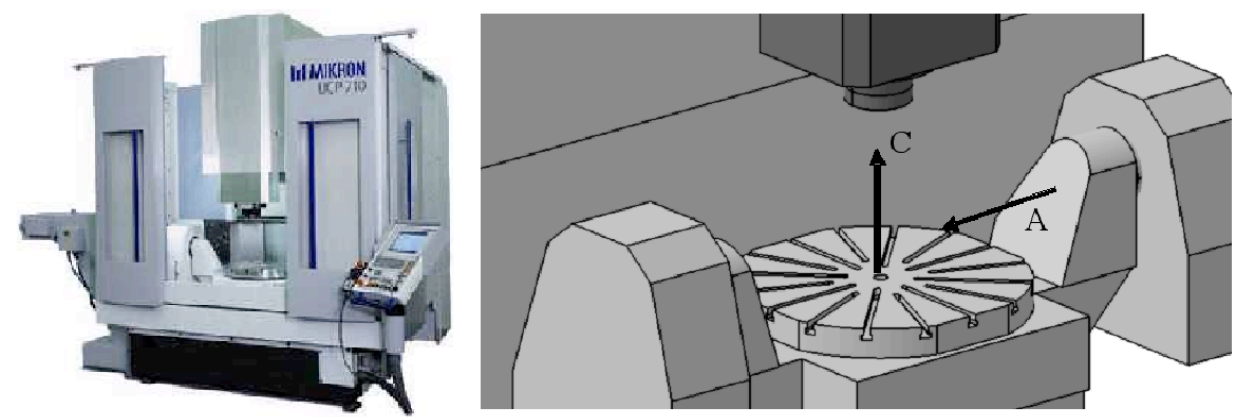

Figure 8. 5-axis machine tool (RRTTT structure)

Hence, the basic issue of discrete 5-axis machining can be formulated as follows: given the point cloud and the 5-axis machine tool, find the minimum number of tool orientations so that all the points are accessible. Actually, this is equivalent to a machining area-by-area problem, for which the data need to be partitioned based on their accessibility (Teng et al, 2006). The method developed consists of two 
main stages: topological and technological data segmentation, and tool path generation. During the segmentation stage, data are partitioned based on accessibility criteria to form groups of points that will be machined with the same tool orientation. Once, points are grouped, algorithms developed in section 2 are applied to generate tool trajectories for both roughing and finishing operations.

\subsection{Data processing for area-by-area machining}

For most of the algorithms developed, a crucial issue is the calculation of geometrical differential properties on discrete data. Indeed, the loss of continuity and the lack of data structure involve that these properties can only be estimated. The major problem concerns in particular the calculation of the local normal. Many authors have investigated this issue (Meek et al. 2000) (Park et al. 2006). As for machining, the normal is simply evaluated from a voxelisation of the data (see $\S 2$ ). For each voxel, the normal is calculated as the normal to the plane that best-fits the points included in the considered voxel. The calculated normal can be interpreted as a local normal, representative of a small portion of the surface (a small sub-set of points). The size of an elementary cell is fixed in function of the free form surface geometry and of the dimension of the tool radius used for the milling. This method is efficient as long as the point number allows the calculation of the least-square plane. When the voxel number is not sufficient (generally, when located at the vicinity of boundaries) the normal is calculated using the voxel and its 27 neighbours. At final, a voxel-map is created: a set of triplets (voxel, normal, barycentre). This voxel-map acts as a filtering of the data.

\subsection{Global visibility}

The computation of tool accessibility at each point is divided into two steps: calculation of the local tool visibility followed by the calculation of the global visibility.

The visibility is evaluated at each point of the voxel-map (each barycentre). A visibility cone is built, which axis is defined by the normal, and the angle of which is directly linked to the tool geometry. Let us denote, $v$ the cone angle: $v=0^{\circ}$ for cylindrical tool, $v=90^{\circ}$ for a ball-end cutter tool (or hemispherical tool) and, $0<v<90^{\circ}$ for a filleted-end tool. In the paper, only the case of the hemispherical tool is investigated (Figure 9). In order to improve the efficiency of the cutting during machining, the cutting zone of the tool is reduced; tool axis orientations parallel to the normal or perpendicular are forbidden. This reduces the visibility zone of the tool as shown in figure 9, which means the possible orientations of the tool relatively to the surface normal. This visibility is thus called tool visibility or local visibility. 


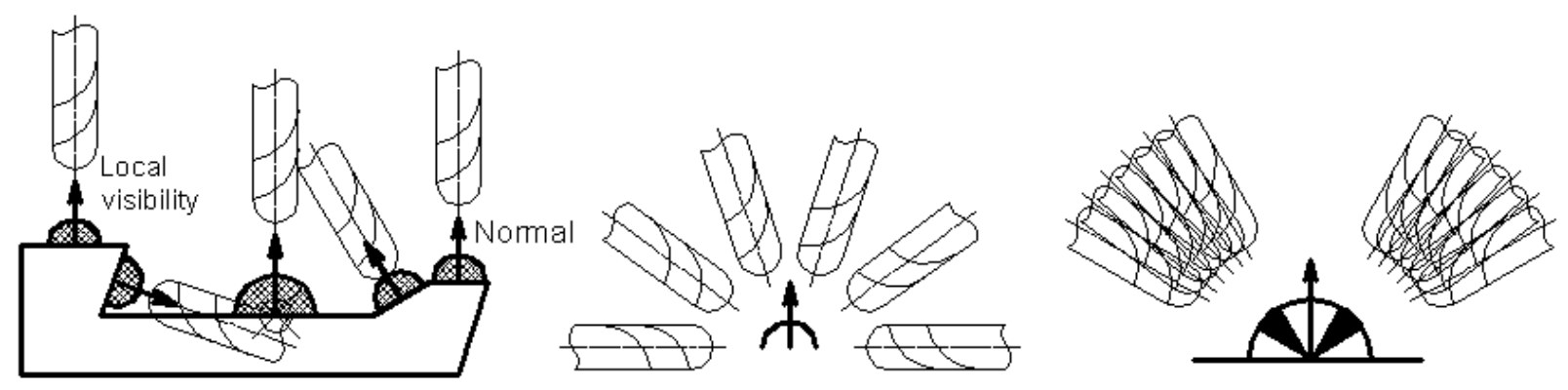

Figure 9. The local visibility

a- Definition of the local visibility

b- Visibility for a hemispherical tool

c- Reduced visibility zone

Anyway, it is important to notice that as points result from a digitizing operation, they are necessarily visible according to the sensor direction that is used to collect them. Although these directions may not be optimal as regards cutting efficiency, they provide a good process initialization.

Once local visibility is calculated, it is necessary to check that the tool body is not in interference with the cloud of points. The verification is performed only for the tool body, without considering the cutting zone of the tool. Indeed, by definition the positioning method is interference-free (see $\S 2.2$ ). To simplify calculations, the tool body is sampled. At final, this provides for each point, tool configurations that are not in interference with the point cloud.

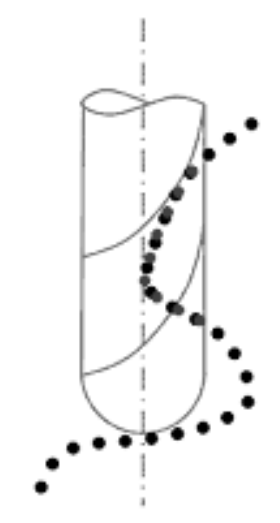

Figure 10. Interference between the tool body and the point cloud

In a last step, machine accessibility must be verified as it is essential to check that each direction of global visibility is included within the visibility space of the machine tool. If not, the tool 
orientation can not be considered as defining a possible set-up. Machine accessibility requires the geometric model of the machine to be known. This last step provides the global visibility.

\subsection{Segmentation for area-by-area machining}

The point cloud segmentation for area-by-area machining, must provide all the set-ups allowing the complete surface machining; it is thus a key point. The efficiency of the approach relies on the use of a visibility matrix (Figure 11). Rows of the matrix correspond to the voxel-map points, and its columns are tool orientations obtained from the visibility step. The value is 1 or 0 , whether the point is visible or not according to the given orientation. The column with the greater number of "1" is first retained, and defines the first set-up. Corresponding points are removed from the visibility matrix, and the search continues as follows:

- Find the column with the maximum number of "1",

- Check that the number of points is greater than a given threshold,

- Check that the number of set-ups is less than a given threshold,

- Group the corresponding points to form a sub-set associated to the given set-up

- Remove those points from the matrix

Once, all the set-ups are found according to the previous criteria, isolated points or small point sub-sets are investigated. Most generally, the algorithm attempts to integrate them to neighbour sub-sets.

$\begin{array}{ccccccc} & \text { ori_1 } & \text { ori_2 } & \text { ori_3 } & \text { ori_4 } & \text { ori_5 } & \text { ori_x } \\ \text { point1 } & 0 & 0 & 0 & 1 & 0 & 1 \\ \text { point2 } & 0 & 1 & 0 & 1 & 0 & 1 \\ \text { point3 } & 0 & 0 & 1 & 1 & 1 & 1 \\ \text { point } 4 & 1 & 1 & 1 & 1 & 0 & 1 \\ \text { point } n-1 & 1 & 0 & 0 & 0 & 0 & 1 \\ \text { point } n & 1 & 1 & 0 & 0 & 1 & 0\end{array}$

Figure 11. Matrix of visibility

\section{$4 \quad$ First application}

The proposed approach is applied for the duplication of a little figurine representing a cartoon character (Figure 13).

The scanning of the object is carried out thanks to a laser plane sensor mounted on a CMM as discussed in $\S 2.3$. Sensor configurations are chosen to guarantee the completeness of the digitized 
points as regards the whole object surface (Mehdi-Souzani et al, 2006). Five sensor directions are used; $\mathrm{z}, \mathrm{x},-\mathrm{x}, \mathrm{y},-\mathrm{y}$. The resulting point cloud is strongly dense and inhomogeneous. The voxel-map is thus built from the points, with a cell size of $2 \mathrm{~mm}$ giving 1437 voxels (Figure 13).

To simplify the problem in this first application, the 5 sensors orientations are considered as possible tool orientations. Corresponding orientations on the machine tool are calculated: $(A=0, C=0)$, $(A=90, C=-90),(A=90, C=90),(A=90, C=180),(A=90, C=0)$. Therefore, for each one, local and global visibilities are checked, with a cone angle equal to $60^{\circ}$. As all the 5 orientations are validated, the point cloud is segmented into 5 areas, defining 5 point sub-sets.

For each set-up, or each point sub-set, the tool-path is calculated according to algorithms developed in section 2.3. The machining zone is defined as follows:

- For each set-up, define a plane perpendicular to the tool axis,

- Project all the voxel vertices that belong to the considered point sub-set; the limits of the rectangular grid are created (Figure 12).

- For each node, if the tool axis intersects the voxels, calculate the Cutter Location point.

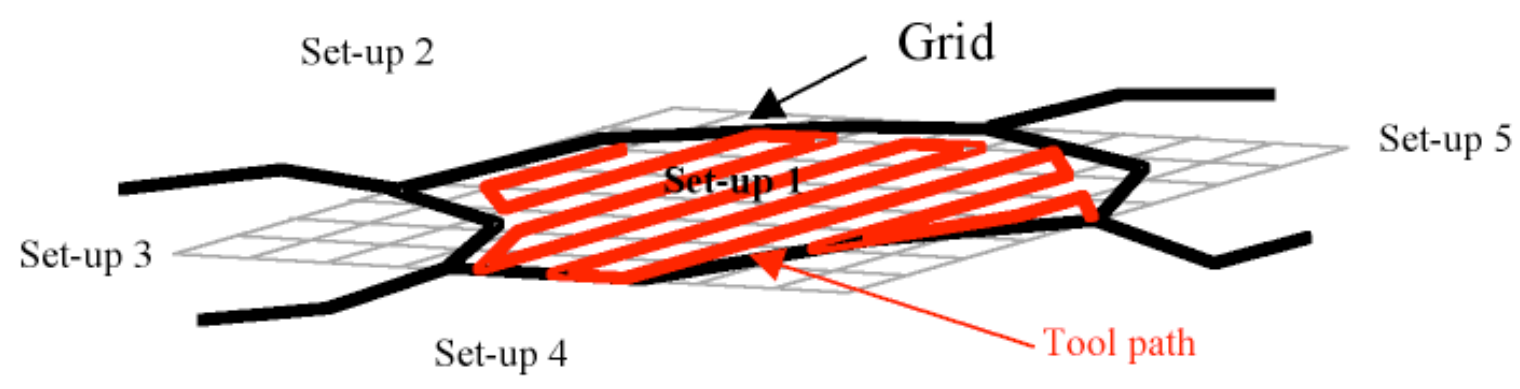

Figure 12. Machining area by set-up

At final, the part machining is carried out on the machine MIKRON UCP 710. Figure 13 shows the result of the milling, which includes both roughing and finishing operations. Due to the limit of the tool radius, some surfaces can not be machined such as the neck of the little character. Nevertheless, some details like the eyes or the wind-breaker buttons are visible which brings out the efficiency of this approach. Although the use of sensor orientations allows limiting the number of part set-ups, the result is not optimized as regards overlapping areas. Some geometrical defects are clearly visible at the machining area junctions, probably due to re-positioning errors on the machine-tool (Figure 14). 


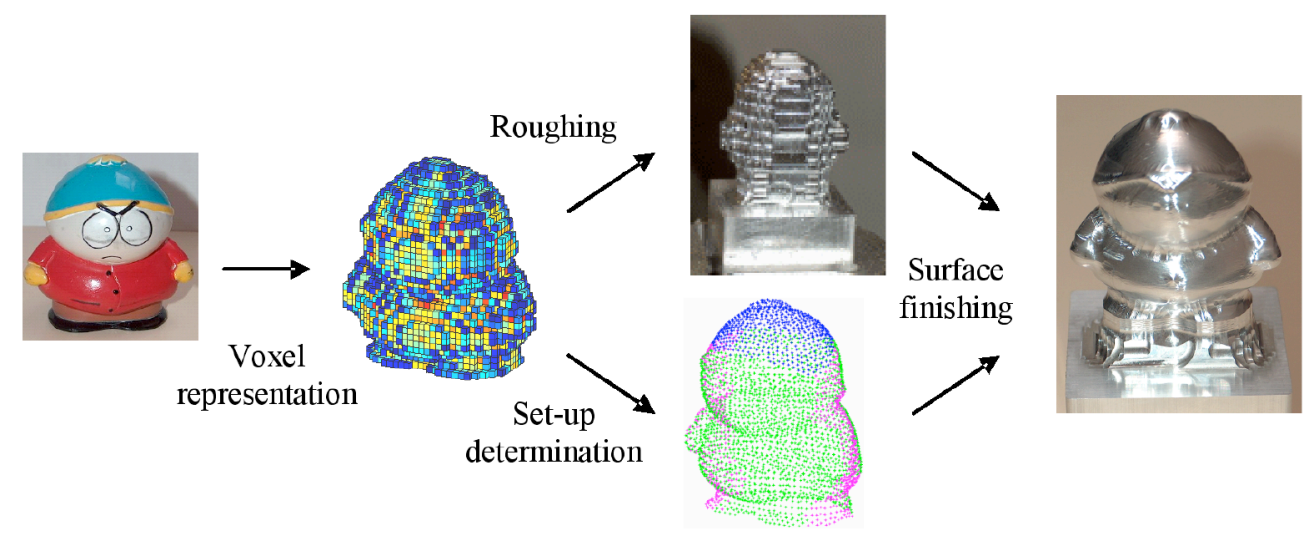

Figure 13. Direct duplication in 5-axis discrete machining.

Those defects are related to machine tool geometric errors (axis straightness, axis perpendicularity...). Although nowadays machine tools have high-performance, geometric errors still remain. Some authors propose to optimize the part set-up in order to minimize those errors (Anotaipaiboon et al, 2006). Other works address the issue of trajectory compensation in order to remove kinematics errors (Mir et al, 2002). Some tests performed on the Mikron UCP shown that registration errors related to tool orientation changes may be close to $0.1 \mathrm{~mm}$ in some cases. Additional tests must be performed in order to provide kinematics errors of the considered machine tool. Therefore, overlaps must be limited which adds a constraint to data segmentation.
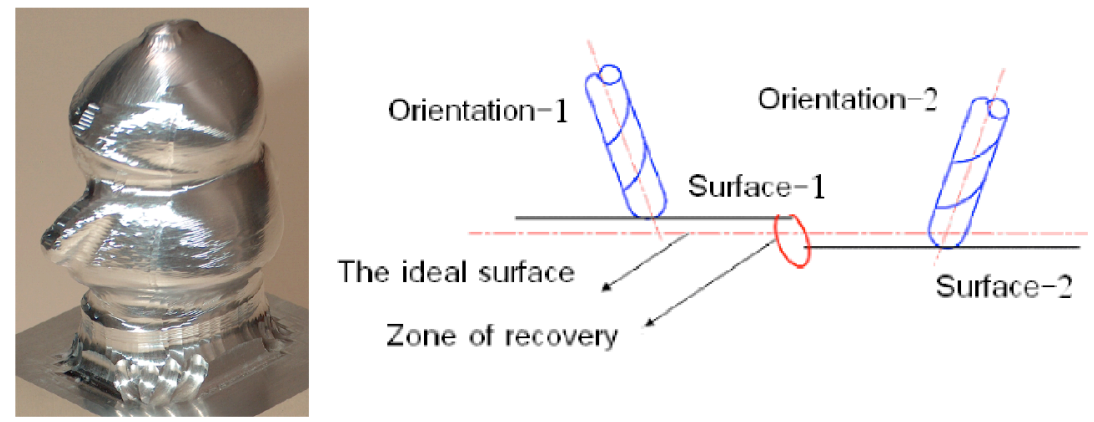

Figure 14. Overlapping defects on the figurine

So, the issue is: finding the best segmentation of the point cloud into sub-sets so that the overlapping length is minimal. The associated optimization problem is hence the following:

- Find the tool orientations (part set-ups)

- So that: the overlapping length is minimal

- Respecting: - local visibility of the tool

- global visibility of the tool and machine accessibility

- minimum number of points to define a machining area 
- maximum number of set-ups

Overlapping zones can be identified as the boundaries of the machining areas. Taking advantages of the voxel-map, these zones can be identified as the voxels; voxels defining the contour of the area constitute the overlapping line. The investigation of the optimisation problem is in progress.

\section{Conclusion}

In the paper, a novel method for duplication of physical models is proposed. The aim is to produce a copy of an existing object which has been digitized using non- contact devices. The step of digitizing generally provides a large cloud of points, dense, noisy and inhomogeneous. To avoid critical issues linked to the RE stage, direct machining of the point cloud is implemented. Algorithms developed for 3-axis ball end milling, for roughing and finishing operations are detailed. The efficiency of direct machining is shown thanks to a simple example. The comparison done with classical methods based on RE highlights the efficiency of the approach in terms of geometrical conformity to the initial surface object. An extension to discrete 5-axis machining is carried out to increase the diversity of forms that can be machined. The main problem is the point cloud segmentation in areas that can be machine with the same tool orientation, defining then a part set-up. To solve this difficulty, a visibility matrix is built. For each point, possible tool orientations, corresponding to part set-ups, are identified according to visibility and accessibility criteria. Points that can be machined with the same tool orientation are grouped to form a point sub-set. Each point sub-set is afterwards machined in 3-axis machining according to algorithms previously developed. The feasibility of direct duplication in 5 axis machining is proved through the duplication of a little figurine. The solution is yet not optimized, and some improvements must be done in particular concerning the overlapping areas.

\section{References}

Anotaipaiboon W., Makhanov S.S., Bohez E.L.J., 2006, Optimal setup for five-axis machining International Journal of Machine Tools and Manufacture, 46(9), 964-977

Breteau P., Thiébaut F., Bourdet P., Lartigue C., 2006, Towards an approach for rapid copying of freeform surfaces in 5-axis machining, 6th International Conference on Integrated Design and Manufacturing in Mechanical Engineering, Grenoble (France), 17-19 May, cdrom paper 
Chen C. Z., Dong Z., Vickers W.G., 2003, Automated surface subdivision and tool path generation for $31 / 21 / 2$ axis CNC machining of sculptured parts, Computers in Industry, 50, 319-331

Chui K. L., Chiu W. K., Yu K. M, 2008, Direct 5-axis tool-path generation from point cloud input using 3D biarc fitting, Robot. Comput.-Integr. Manuf., 24(2), 270-286

Feng, H-Y., Teng, Z., 2005, Iso-planar piecewise linear NC tool path generation from discrete measured data points, Computer-Aided Design, 37, 55-64

Jerard, R., Hussaini, S., Drysdale, R., Schaudt B., 1989 Approximate methods for simulation and verification of numerically controlled machining programs. The Visual Computer, 5, 329-348

Lin, A-C, Liu, H-T, 1998, Automatic generation of NC cutter path from massive data points, Computer-Aided Design, 30, 77-90

Meek D.S., Walton D.J., 2000 On surface normal and Gaussian curvature approximations given data sampled from a smooth surface, Computer Aided geometric Design, 17, 521-543.

Mehdi-Souzani, C., Thiébaut F., Lartigue, C., 2006, Scan planning strategy for a general digitized surface, Journal of Computing \& Information Science in Engineering, ASME International, 6(4), 331339

Mir, Y.A., Mayer, J.R.R., Fortin C., 2002, Tool path error prediction of a five axis machine tool with geometric errors», J. of Engineering Manufacture, 216(5), 697-712

Osty, G., Contri, A., Duc, E., 2000, Free-form copying from point clouds; A tool-path calculation method using B-Spline interpolation, RCFAO, Rapid production, 367-382

Ou Yang, D., Van Nest B.A., Feng, H-Y, 2005, Determining gouge-free ball-end mills for 3D surface machining from point cloud data, Robotics and Computer-Integrated Manufacturing, 21, 338-345

Park S-C., Chung, S-C, 2003, Tool-path generation from measured data, Computer-Aided Design, 35, 467-475 
Park J.C., Shin H., Choi B.K., 2006, Elliptic Gabriel graph for finding neighbors in a point set and its application to normal vector estimation, Computer-Aided Design, 38(6), 619-626

Peng Y.H., Yin, .W., 2007, Direct tool path regeneration for physical object modification from digitized points in reverse engineering, International Journal of Manufacturing Technology, 33, 12041211

Suzuki, H., Kuroda Y., Sakamoto M., Haramaki S., Van Brussel H., 1991, Development of the CAD/CAM System based on parallel processing and inverse offset method, Transputing'91 Proceeding of the world Transputer user Group (WOTUG) conference

Teng Z., feng H-S, Azeem, A, 2006, generating tool paths from point cloud data via machining area segmentation, International Journal of Manufacturing Technology, 30, 254-260

Varady T., Martin RR, Cox J., 1997, Reverse Engineering of geometric models - an introduction, Computer-Aided design, 29(4), 255-268 